\title{
DEFORMED HARISH-CHANDRA HOMOMORPHISM FOR THE CYCLIC QUIVER
}

\author{
Alexei Oblomkov
}

\begin{abstract}
In the case of cyclic quiver we prove that the deformed Harish-Chandra map whose existence was conjectured by Etingof and Ginzburg is well defined. As an application we prove a Kirillov-type formula for the cyclotomic Bessel function.
\end{abstract}

\section{Introduction}

In this note we prove the first part of Conjecture 11.22 from the paper [EG] on the deformed Harish-Chandra isomorphism for the cyclic quiver. In other words, we prove that the deformed Harish-Chandra homomorphism is well defined. The constructed Harish-Chandra homomorphism can be used to study the representation theory of the rational Cherednik algebras associated to the complex reflection group $\mathfrak{S}_{n} \ltimes\left(\mathbb{Z}_{\ell}\right)^{n}$ where $\mathfrak{S}_{n}$ is the symmetric group [Ch, BEG, BEG1, E, GG]. In particular, the paper $[G]$ explains the construction for the shift functor. Also the last paper proves the second part of the Conjecture 11.2 concerning the kernel of the deformed HarishChandra homomorphism.

The structure of the text is as follows. In subsections 2.1,2.2 we define the main objects: the cyclic quiver $Q$ along with the space of representations $R Q_{n}$ of the associated quiver algebra $\mathbb{C} Q$, and the rational Cherednik algebra $H_{n}(k, c)$ together with its spherical subalgebra $H_{n}^{s p h}(k, c)$. In subsections 2.3,2.4 we define the Dunkl operator embedding $\Theta_{k, c}$ (and $\Theta_{k, c}^{s p h}:=\left.\Theta_{k, c}\right|_{H_{n}^{s p h}(k, c)}$ ) and the radial part map $\mathfrak{R}_{k, c}$ along with its twisted version $\Re_{k, c}^{t w}$. In subsection 2.5 the main theorem is formulated. It states that the images of $\Theta_{k, c}^{s p h}$ and $\mathfrak{R}_{k, c}^{t w}$ are equal. Section 3 is devoted to the proof of the theorem. In subsection 4.1 of Section 4 we give a definition of the cyclotomic Bessel function and in the subsection 4.3 we prove an integral formula for this function. The formula generalizes the well known interpretation of the classical Bessel function as the integral over two dimensional sphere [V]. In subsection 4.6 we relate our integral formula to the theory of spherical functions and Kirillov's orbit method.

The idea of the proof is to establish the equality $\left.\operatorname{Im} \Theta_{k, c}^{s p h}=\operatorname{Im} \Re_{k, c}^{t w} 1\right)$ after localization by $x_{i}=0,1 \leq i \leq n$ and 2) after localization by $x_{i}^{\ell}=x_{j}^{\ell}, 1 \leq i<j \leq n$. These two statements imply the theorem. Statement 1 ) is checked using the result for $\ell=1$ proved in [EG] and 2) is proved using the result for $n=1$ proved by Holland [Ho].

Received by the editors May 27, 2005.

This work was partially supported by the NSF grant DMS-9988796 and CRDF grant RM1-2545MO-03. 


\section{The main theorem}

2.1. Quiver $Q$. Let $Q$ be the cyclic quiver with $\ell$ arrows oriented clockwise. We label the vertices of the quiver by numbers $0, \ldots, \ell-1$ in the clockwise direction. Let $R Q_{n}$ be the space of representations of the associated quiver algebra $\mathbb{C} Q$ of dimension $n \delta$ where $\delta=(1, \ldots, 1)$. In other words, $R Q_{n}$ is the vector space $\oplus_{i=0}^{\ell-1} \operatorname{Hom}\left(V_{i+1}, V_{i}\right)$, where $V_{i}$ is the $n$-dimensional vector space assigned to the vertex $i$, and $V_{\ell}=V_{0}$. Let us denote by $A_{i, i+1}$ the elements of $\operatorname{Hom}\left(V_{i+1}, V_{i}\right)$.

There is a natural action of the group $G_{n}=\prod_{i=0}^{\ell-1} G L\left(V_{i}\right)$ on the space $R Q_{n}$ by conjugation. If $g=\left(g_{0}, \ldots, g_{\ell-1}\right) \in G, g_{i} \in G L\left(V_{i}\right)$ and $A=\left(A_{0,1}, \ldots, A_{\ell-1,0}\right)$ then $g \cdot A=\left(g_{0} A_{0,1} g_{1}^{-1}, g_{1} A_{1,2} g_{2}^{-1}, \ldots, g_{\ell-1} A_{\ell-1,0} g_{0}^{-1}\right)$. Because the element $\left(c I d_{n}, \ldots, c I d_{n}\right)$ acts trivially on $R Q_{n}$, we actually have an action of the group $P G_{n}=$ $G_{n} / \mathbb{C}^{*}$ on $R Q_{n}$. We use the notation $\mathfrak{p g}_{n}$ for the Lie algebra of $P G_{n}$.

2.2. The rational Cherednik algebra $H_{n}(k, c)$. We denote by $\Gamma$ the cyclic group $\mathbb{Z}_{\ell}$ and by $\Gamma_{n}$ the semidirect product $\mathfrak{S}_{n} \ltimes \Gamma^{n}$ with the symmetric group $\mathfrak{S}_{n}$. The group $\Gamma$ is generated by an element $\alpha$ and the group $\mathfrak{S}_{n}$ is generated by transpositions $s_{i j}$ exchanging $i$ and $j, i \neq j$. We denote by $\gamma_{i}$ the element of $\Gamma^{n}$ which is equal to $\gamma$ at the $i$-th place and 1 at the other places. Then the conjugation by the element $s_{i j}$ of the subgroup $\mathfrak{S}_{n} \subset \Gamma_{n}$ acts on the normal subgroup $\Gamma^{n}$ by the formula:

$$
s_{i j} \alpha_{i}^{s} s_{i j}=\alpha_{j}^{s}, \quad s_{i j} \alpha_{p}^{s} s_{i j}=\alpha_{p}^{s}, \quad p \neq i, j .
$$

Let $L$ be a two dimensional vector space and fix a basis $x, y$ in $L$. Denote by $L_{n}$ the vector space $L^{\oplus n}$, and let $x_{i}, y_{i}$ denote $x, y$ in the $i$-th component of the sum. The group $\Gamma_{n}$ acts on $L_{n}$ :

$$
\begin{gathered}
\alpha_{i}\left(x_{i}\right)=\epsilon x_{i}, \quad \alpha_{i}\left(x_{j}\right)=x_{j}, \quad \alpha_{i}\left(y_{i}\right)=\epsilon^{-1} y_{i}, \quad \alpha_{i}\left(x_{j}\right)=x_{j}, \\
s_{i j}\left(x_{i}\right)=x_{j}, \quad s_{i j}\left(y_{i}\right)=y_{j} \\
s_{i j}\left(x_{k}\right)=x_{k}, \quad s_{i j}\left(y_{k}\right)=y_{k}, \quad 1 \leq i, j \neq k \leq n
\end{gathered}
$$

where $\epsilon$ is a (fixed) primitive $\ell$-th root of unity.

Let $T L_{n}$ be the tensor algebra of $L_{n}$, that is the free algebra generated by $x_{i}, y_{i}$, $i=1, \ldots, n$. There is an action of $\Gamma_{n}$ on the algebra $T L_{n}$, hence we can form a smash product $\Gamma_{n} \ltimes T L_{n}$.

Definition. [EG] The algebra $H_{n}(k, c), k \in \mathbb{C}, c \in \mathbb{C}^{\ell-1}$ is the quotient of the algebra $\Gamma_{n} \ltimes T L_{n}$ by the relations:

$$
\begin{gathered}
{\left[x_{i}, x_{j}\right]=0, \quad\left[y_{i}, y_{j}\right]=0, \quad 1 \leq i, j \leq n,} \\
{\left[y_{i}, x_{i}\right]=1-k \sum_{j \neq i} \sum_{m=0}^{\ell-1} s_{i j} \alpha_{i}^{m} \alpha_{j}^{-m}+\sum_{m=1}^{\ell-1} c_{m} \alpha_{i}^{m}, \quad 1 \leq i \leq n,} \\
{\left[y_{i}, x_{j}\right]=k \sum_{m=0}^{\ell-1} s_{i j} \epsilon^{m} \alpha_{i}^{m} \alpha_{j}^{-m}, \quad 1 \leq i \neq j \leq n .}
\end{gathered}
$$

If $\mathrm{e} \in \mathbb{C}\left[\Gamma_{n}\right]$ is the idempotent corresponding to the trivial representation then $H_{n}^{s p h}(k, c)=\mathrm{e} H_{n}(k, c)$ e is called the spherical subalgebra of $H_{n}(k, c)$. 
2.3. Dunkl operators. From the definition of $H_{n}(k, c)$ we see that the subalgebra generated by $x_{i}, i=1, \ldots, n$ is the algebra of polynomials of $n$ variables. Thus we can think of $x_{i}, i=1, \ldots, n$ as functions on the $n$-dimensional space $\mathfrak{t}=\mathbb{C}^{n}$. Let us denote by $\mathfrak{t}^{r e g}$ the open subset of $\mathfrak{t}$ given by the conditions $x_{i} \neq \epsilon^{s} x_{j}$ and $x_{i} \neq 0$ for $1 \leq i \neq j \leq n, s=0, \ldots, \ell-1$.

Let us denote by $\mathrm{D}\left(\mathfrak{t}^{r e g}\right)$ the algebra of differential operators on $\mathfrak{t}^{r e g}$ and let $\Gamma_{n} \ltimes$ $\mathrm{D}\left(\mathfrak{t}^{r e g}\right)$ denote the smash product. As explained in [DO] there is an embedding $\Theta_{k, c}$ of the algebra $H_{n}(k, c)$ into $\Gamma_{n} \ltimes \mathrm{D}\left(\mathfrak{t}^{r e g}\right)$. The embedding is given by the formulas

$$
\begin{gathered}
x_{i} \mapsto x_{i}, \quad \Gamma_{n} \ni g \mapsto g, \quad y_{i} \mapsto \mathcal{D}_{i}, \\
\mathcal{D}_{i}=\frac{\partial}{\partial x_{i}}+k \sum_{j \neq i} \sum_{m=0}^{\ell-1} \frac{1}{x_{i}-\epsilon^{m} x_{j}}\left(s_{i j} \alpha_{i}^{m} \alpha_{j}^{-m}-1\right)+\sum_{m=1}^{\ell-1} \frac{c_{m}}{\left(\epsilon^{m}-1\right) x_{i}}\left(\alpha_{i}^{m}-1\right) .
\end{gathered}
$$

The map $\Theta_{k, c}$ induces a map from the spherical subalgebra $H_{n}^{s p h}(k, c)$ to the algebra $\mathrm{D}\left(\mathfrak{t}^{r e g}\right)^{\Gamma_{n}}$ of the $\Gamma_{n}$-invariant differential operators on $\mathfrak{t}^{r e g}$. We use the letter $\Theta_{k, c}^{s p h}$ for this map.

2.4. The radial part map. Let us define a character $\chi_{c}$ of $\mathfrak{p g}_{n}$ by the formula

$$
\chi_{c}(x)=\sum_{i=0}^{\ell-1} C_{i} \operatorname{Tr}\left(x_{i}\right),
$$

where $C_{i}=\frac{1}{\ell}-\frac{1}{\ell} \sum_{m=1}^{\ell-1} \epsilon^{m i} c_{m}$ for $i=1, \ldots, \ell-1$ and $C_{0}=\frac{1-\ell}{\ell}-\frac{1}{\ell} \sum_{m=1}^{\ell-1} c_{m}$. We use the same letter for the one dimensional representation of $P G_{n}$.

Let pr: $G_{n} \rightarrow P G L\left(V_{0}\right)$ be a projection of $G_{n}$ onto the 0-th component composed with the map $G L\left(V_{0}\right) \rightarrow P G L\left(V_{0}\right)$. Let $k \in \mathbb{Z}_{+}, W_{k} \subset \mathbb{C}\left[y_{1}, \ldots, y_{n}\right]$ is the subspace of the homogeneous polynomials of degree $k n$. Let $\rho_{k}: \mathfrak{s l}\left(V_{0}\right) \rightarrow \mathfrak{g l}\left(W_{k}\right)$ be the corresponding Lie algebra map. We denote by the same letter $\rho_{k}$ the representation of $\mathfrak{p g}_{n}$ induced by the projection $p r$. We use the notation $\rho_{k, c}$ for $\rho_{k} \otimes \chi_{c}$.

To define the radial part map we need the space of $\mathfrak{p g}_{n}$-equivariant vector valued functions $F u n_{k, c}$. Let $F u n^{\prime}$ be the space of the functions on $R Q_{n}$ of the form $f=$ $\tilde{f} \prod_{i=0}^{\ell-1}\left(\operatorname{det}\left(A_{i, i+1}\right)\right)^{r_{i}}$ where $\tilde{f}$ is a rational function on $R Q_{n}$ and $r_{i}=-\sigma+\sum_{j=0}^{i} C_{j}$, $i=0, \ldots, \ell-1, \sigma:=\sum_{s=0}^{\ell-1} s C_{s} / \ell$. The function $f \in F u n^{\prime} \otimes W_{k}$ is from the space $F_{k, c}$ if and only if $L_{g}(f)(x)=\left.\frac{d f\left(x e^{g t}\right)}{d t}\right|_{t=0}=\rho_{k, c}(g) f(x)$ for all $g \in \mathfrak{p g}_{n}, x \in R Q_{n} .{ }^{1}$

If $x \in \mathbb{C}^{n}$ is a vector then denote by $\operatorname{diag}(x)$ the diagonal matrix of size $n$. Let us denote by $\Delta$ the map $\mathbb{C}^{n} \rightarrow R Q_{n}$ which sends $x$ to the element $(\operatorname{diag}(x), \ldots, \operatorname{diag}(x))$. The image $\mathcal{S}$ of $\Delta$ is a slice for the action of $P G_{n}$ on $R Q_{n}$. That is, for a generic element $A \in R Q_{n}$ there exists an element $g \in P G_{n}$ such that $g \cdot A=\Delta(x) \in \mathcal{S}$. Also, it is easy to see that the element $x$ is unique up to the action of $\Gamma_{n}$.

The zero weight space $W_{k}[0]$ is one dimensional and, the restriction of $f \in F u n_{k, c}$ to $\mathcal{S}$ takes values in $W_{k}[0]$. That is, the restriction $\operatorname{Res}(f)$ of the function $f$ can be regarded as scalar function. Moreover, $f$ is uniquely determined by its restriction $\operatorname{Res}(f)$, and if $\bar{f}$ is $\Gamma_{n}$-equivariant then there exists a function $f \in F u n_{k, c}$ such that

\footnotetext{
${ }^{1}$ The fact the functions from $F u n_{k, c}$ are multivalued functions on $R Q_{n}$ does not create the problem for us because for the computation of the radial part we only need the restriction of the function to a small neighborhood of the slice $\mathcal{S}$ defined below.
} 
$\bar{f}=R e s(f)$. Thus we can define the radial part map $\mathfrak{R}_{k, c}: \mathrm{D}\left(R Q_{n}\right)^{\mathfrak{p g} \mathfrak{g}_{n}} \rightarrow \mathrm{D}\left(\mathfrak{t}^{\text {reg }}\right)$ by the condition:

$$
\operatorname{Res}(D(f))=\mathfrak{R}_{k, c}(D) \operatorname{Res}(f),
$$

for all $f \in F_{n_{k, c}}$. Here we used the notation $\mathrm{D}\left(R Q_{n}\right)$ for the space of the differential operators on $R Q_{n}$. It is easy to see that in fact this map lands in the subspace $\mathrm{D}\left(R Q_{n}\right)^{\mathfrak{p g}_{n}}$ of $\mathfrak{p g}_{n}$-invariant differential operators on $R Q_{n}$. In particular, for a function $h \in \mathbb{C}\left[R Q_{n}\right]^{\mathfrak{p g}_{n}}$ we have $\mathfrak{R}_{k, c}(h)=\operatorname{Res}(h)$.

Remark 2.1. As explained at [EG] one can generalize the definition of $\mathfrak{R}_{k, c}$ to the case of any $k \in \mathbb{C}$. Namely, in this one should consider the representation $\tilde{W}_{k}=$ $\left(y_{1} \ldots y_{n}\right)^{k} \mathbb{C}_{(0)}\left[y_{1}^{ \pm 1}, \ldots, y_{n}^{ \pm 1}\right]$ where $\mathbb{C}_{(0)}\left[y_{1}^{ \pm 1}, \ldots, y_{n}^{ \pm 1}\right]$ is the space of the Laurent polynomials of degree 0 . If $k$ is a positive integer then $W_{k}$ is a subrepresentation of $\tilde{W}_{k}$, so the two settings are equivalent.

It is more convenient to use the twisted version $\mathfrak{R}^{t w}$ of the radial part map:

$$
\begin{gathered}
\mathfrak{R}_{k, c}^{t w}(D):=\delta_{k, c}^{-1} \circ \mathfrak{R}(D) \circ \delta_{k, c}, \\
\delta_{k, c}(x):=\delta^{k+1} \delta_{\Gamma}^{\ell \sigma}, \quad \delta_{\Gamma}:=\prod_{i=1}^{n} x_{i}, \quad \delta=\prod_{1 \leq i<j \leq n}\left(x_{i}^{\ell}-x_{j}^{\ell}\right) .
\end{gathered}
$$

\subsection{Main result.}

Theorem. For all values of $k, c$ we have $\operatorname{Im} \Re_{k, c}^{t w}=\operatorname{Im} \Theta_{k, c}^{s p h}$.

When $n=1$ the theorem is a particular case of the results of Holland [Ho], and when $\ell=1$ the theorem is proved by Etingof and Ginzburg [EG] who also conjectured the statement of the theorem for the general $\ell$. The map $\mathfrak{H C}_{k, c}:=\mathfrak{R}_{k, c}^{t w} \circ \Theta_{k, c}^{-1}$ : $\mathrm{D}\left(R Q_{n}\right)^{\mathfrak{p g}_{n}} \rightarrow \mathrm{e} H_{n}(k, c)$ e whose existence follows from the theorem is called the deformed Harish-Chandra homomorphism [EG].

\section{Proofs}

From the definition of $H_{n}(k, c)$ we see that we can localize our algebras $H_{n}(k, c)$ by inverting a polynomial of $x_{i}$ which is preserved by the action of $\Gamma_{n}$. For example, the polynomials $\delta, \delta_{\Gamma}$ have this property.

Let us also introduce the notation $H_{n}(k)$ for the rational Cherednik algebra in the case $\ell=1$. This algebra is the quotient of $\mathfrak{S}_{n} \ltimes \mathbb{C}\left\langle X_{1}, Y_{1}, \ldots, X_{n}, Y_{n}\right\rangle$ by the relations:

$$
\begin{gathered}
{\left[Y_{i}, Y_{j}\right]=\left[X_{i}, X_{j}\right]=0, \quad 1 \leq i, j \leq n,} \\
{\left[Y_{i}, X_{j}\right]=k s_{i j}, \quad 1 \leq i \neq j \leq n,} \\
{\left[Y_{i}, X_{i}\right]=1-k \sum_{j \neq i} s_{i j} .}
\end{gathered}
$$

Then we have the following propositions about the localizations.

Proposition 3.1. If $\mathrm{e}_{\Gamma} \in \mathbb{C}\left[\Gamma^{n}\right] \subset \mathbb{C}\left[\Gamma_{n}\right]$ is the idempotent corresponding to the trivial representation, and $H_{n}(k)_{(X)}$ is the localization by the variables $X_{i}$ then we have the following isomorphism

$$
\left(e_{\Gamma} H_{n}(k, c) e_{\Gamma}\right)_{\delta_{\Gamma}} \simeq H_{n}(k)_{(X)} .
$$


The isomorphism is induced by the embedding $\mathbf{j}: H_{n}(k) \hookrightarrow\left(e_{\Gamma} H_{n}(k, c) e_{\Gamma}\right)_{\delta_{\Gamma}}$ given by:

$$
Y_{i} \mapsto \ell^{-1} x_{i}^{1-\ell} y_{i}, \quad X_{i} \mapsto x_{i}^{\ell}, \quad 1 \leq i \leq n .
$$

Proof. Let $T$ is the $n$ dimensional space with the coordinates $X_{i}=x_{i}^{\ell}, i=1, \ldots, n$ and $T^{r e g}$ is the open subset defined by the conditions $X_{i} \neq X_{j}, X_{i} \neq 0,1 \leq i \neq j \leq n$. We have an obvious isomorphism $\phi: \mathrm{D}\left(T^{r e g}\right) \rtimes \mathfrak{S}_{n} \rightarrow \mathrm{e}_{\Gamma}\left(\mathrm{D}\left(\mathfrak{t}^{r e g}\right) \rtimes \Gamma_{n}\right) \mathrm{e}_{\Gamma}$. It is enough to check that $\Theta_{k, c} \circ \mathbf{j}\left(Y_{i}\right)=\phi \circ \Theta_{k}\left(Y_{i}\right), i=1, \ldots, n$, where $\Theta_{k}$ is the Dunkl embedding for $H_{n}(k)$ (see [EG]). It can be done by a direct computation.

Proposition 3.2. We have an isomorphism $H_{n}(k, c)_{\delta} \simeq H_{n}(0, c)_{\delta}$ induced by the embedding $\mathbf{i}: H_{n}(0, c) \hookrightarrow H_{n}(k, c)_{\delta}$ given by:

$$
y_{i} \mapsto y_{i}+k \sum_{j \neq i} \sum_{m=0}^{\ell-1} \frac{1}{x_{i}-\epsilon^{m} x_{j}}\left(s_{i j} \alpha_{i}^{m} \alpha_{j}^{-m}-1\right), \quad x_{i} \mapsto x_{i}, \quad 1 \leq i \leq n .
$$

Proof. The proof follows from the formulas for the Dunkl operators.

Remark 3.1. If $H_{1}(c)$ is the rational Cherednik algebra for $n=1$ then we have

$$
H_{n}(0, c)=\mathfrak{S}_{n} \ltimes H_{1}(c)^{\otimes n} .
$$

It is shown in the next two lemmas that we can also make compatible localization on the space $R Q_{n}$.

Namely, let $\widehat{R Q_{n}} \subset R Q_{n}$ be the open subset consisting of the points $A \in R Q_{n}$ with the property that the maps $A_{i, i+1}, i=0, \ldots, \ell-1$ are invertible. We have a map $\pi$ : $\widetilde{R Q_{n}} \rightarrow G L_{n}=G L\left(V_{0}\right)$ which sends $A \in \widetilde{R Q_{n}}$ to the product $A_{0,1} A_{1,2} \ldots A_{\ell-1,0}$. Let $\tilde{i}: G L\left(V_{0}\right) \rightarrow \widetilde{R Q_{n}}$ be a section of $\pi: \tilde{i}(X)=(X, 1, \ldots, 1)$. We define a homomorphism

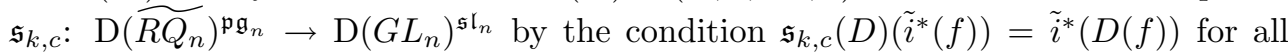
$f \in$ Fun $_{k, c}, D \in \mathrm{D}\left(\widetilde{R Q_{n}}\right)^{\mathfrak{p g}_{n}}$.

As we know $\mathrm{D}\left(G L_{n}\right)^{\mathfrak{s l}_{n}}=\mathrm{D}\left(\mathfrak{g l}_{n}\right)_{\text {loc }}^{\mathfrak{s l}_{n}}$ where the subscript 'loc' stands for localization by the determinant. In particular the construction for the radial part from the previous section gives us a map $\mathfrak{R}_{k}^{t w}: \mathrm{D}\left(\mathfrak{g l}_{n}\right)_{l o c}^{\mathfrak{S l}_{n}} \rightarrow \mathrm{D}\left(T^{r e g}\right)^{\mathfrak{S}_{n}}$. Let us introduce a map $\pi_{t}: \mathfrak{t} \rightarrow T$ defined by $\pi_{t}^{*}\left(X_{i}\right)=x_{i}^{\ell}, i=1, \ldots, n$.

It turns out that we can put all these maps into a commutative diagram:

Lemma 3.1. The diagram

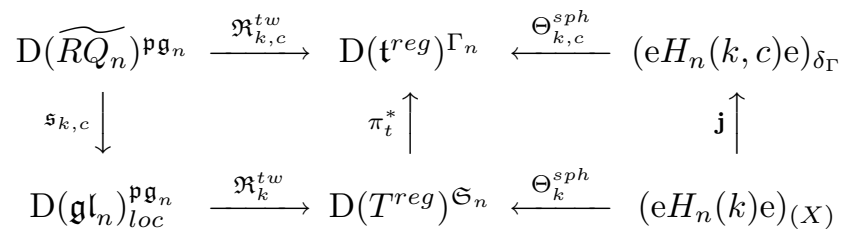

is commutative.

Proof. The map $\pi_{t}^{*}$ acts on the differential operators by the change of variables $X_{i} \mapsto$ $x_{i}^{\ell}, i=1, \ldots, \ell$, hence from the description of the map $\mathbf{j}$ we see that the right half of the diagram is commutative. That the left half of the diagram commutes follows from the definition of the radial part map. 
Let us consider another open subset $R Q_{n}^{0} \subset R Q_{n}$ consisting of points $A \in R Q_{n}^{0}$ such that the matrix $\pi(A)$ is diagonalizable with distinct eigenvalues and matrices $Y_{i}=A_{i, i+1} \ldots A_{i-1, i} \in \operatorname{End}\left(V_{i}\right), i \neq 0$ are nondegenerate. Let $\mathcal{T} \subset R Q_{n}^{0}$ be the subset of diagonal matrices (that is, the matrices $A_{i, i+1}$ are diagonal for $i=0, \ldots, \ell-$ 1) and let us denote by $i$ the embedding of $\mathcal{T}$ into $R Q_{n}^{0}$.

It is elementary to see that $R Q_{n}^{0}=P G_{n}(\mathcal{T})$. Hence $i$ induces a map $i^{*}: \mathrm{D}\left(R Q_{n}^{0}\right)^{\mathfrak{p g}_{n}} \rightarrow \mathrm{D}^{0}$ where $\mathrm{D}^{0}$ is a algebra of differential operators which we describe below. Let $\mathcal{O}_{\mathcal{T}}^{K_{n}}$ be a ring of invariants of $K_{n}=\mathfrak{S}_{n} \ltimes H, H=\left(\mathbb{C}^{*}\right)^{n \ell}$. The differential operators preserving $\mathcal{O}_{\mathcal{T}}^{K_{n}}$ form a subalgebra $\mathrm{D}^{\prime} \subset \mathrm{D}(\mathcal{T})$. The elements of $\mathrm{D}^{\prime}$ also preserve the subspace $F u n_{k, c}^{0}:=F u n_{k, c} \mid \mathcal{T}$ and the homomorphism $r: \mathrm{D}^{\prime} \rightarrow \operatorname{End}_{\mathbb{C}}\left(\right.$ Fun $\left._{k, c}^{0}\right)$ is well defined. The algebra $\mathrm{D}^{0}$ is the image of $r$.

We also have the radial part map $\mathfrak{R}_{c}: \mathrm{D}^{0} \rightarrow \mathrm{D}\left(\mathfrak{t}^{r e g}\right)^{\Gamma_{n}}$. We actually need a twisted version of this map $\mathfrak{R}_{c}^{t w}=\delta_{\Gamma}^{-\ell \sigma} \circ \mathfrak{R}_{c} \circ \delta_{\Gamma}^{\ell \sigma}$.

Lemma 3.2. The diagram

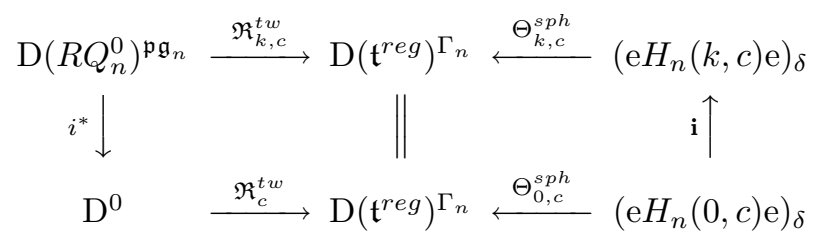

is commutative.

The proof of the lemma is analogous to the proof of the previous lemma.

We also use notations $\mathfrak{R}_{c}^{t w}$ and $\Theta_{c}^{s p h}$ for the radial part and Dunkl operator maps in the case $n=1$. The results of Holland imply the following proposition. It is the simplest case of his theorem and we provide a proof below.

Proposition 3.3. If $n=1$ then $\operatorname{Im} \Re_{c}^{t w}=\operatorname{Im} \Theta_{c}^{s p h}$.

Proof. By the definition of the Dunkl operators, we have $\Theta_{c}^{s p h}\left(\mathrm{e} y^{\ell} \mathrm{e}\right)=D^{\prime}$ where $D^{\prime} \in \mathrm{D}\left(\mathfrak{t}^{r e g}\right)^{\Gamma}$ A simple computation with Dunkl operators shows that:

$$
D^{\prime}=\left(\frac{\partial}{\partial x}+\frac{\tilde{C}_{0}}{x}\right)\left(\frac{\partial}{\partial x}+\frac{\tilde{C}_{0}+\tilde{C}_{1}}{x}\right) \cdots\left(\frac{\partial}{\partial x}+\frac{\tilde{C}_{0}+\cdots+\tilde{C}_{\ell-1}}{x}\right),
$$

where $\tilde{C}_{i}=\sum_{m=1}^{\ell-1} \epsilon^{m i} c_{i}$ The differential operator $D^{\prime}$ acts on the space $F$ spanned by the monomials $x^{r}, r \in \mathbb{C}$ and $D^{\prime}$ is determined (up to a scalar) by the kernel of the action on $F$. It easy to compute this kernel: $\operatorname{ker} D^{\prime}=\left\langle x^{a_{0}}, \ldots, x^{a_{\ell-1}}\right\rangle$ where $a_{i}=-\ell \sum_{s=0}^{i} C_{s}$

The operator

$$
D^{\prime \prime}=\mathfrak{R}_{c}\left(\frac{\partial}{\partial A_{0,1}} \frac{\partial}{\partial A_{1,2}} \cdots \frac{\partial}{\partial A_{\ell-1,0}}\right),
$$

also acts on $F$. The function $f=A_{0,1}^{r_{0,1}} \ldots A_{\ell-1,0}^{r_{\ell-1,0}}$, where $r_{i, i+1}=m / \ell-\sigma+\sum_{s=0}^{i} C_{s}$, has the properties that $f$ is $\chi_{c}$-equivariant and Resf $=x^{m}$. That is, if $m=\ell \sigma+a_{i}$ then the function $f$ does not depend on $A_{i, i+1}$, hence $D^{\prime \prime}(f)=0$. This implies that $\operatorname{ker} D^{\prime \prime}=\left\langle x^{b_{0}}, \ldots, x^{b_{\ell-1}}\right\rangle$ where $b_{i}=\ell \sigma+a_{i}$. 
Because both operators are of order $\ell$ we have proved that $D^{\prime}=x^{-\ell \sigma} \circ D^{\prime \prime} \circ x^{\ell \sigma}$. This implies the inclusion $\operatorname{Im} \Theta_{c}^{s p h} \subset \operatorname{Im} \Re_{c}^{t w}$ because $\mathrm{e} H_{1}(c)$ e is generated by $x^{\ell}$ and $y^{\ell}[\mathrm{LS}]$.

To prove the opposite inclusion we need the filtrations on $H_{1}(c), \mathrm{D}\left(\mathfrak{t}^{r e g}\right)$ and $\mathrm{D}\left(R Q_{n}\right)$. These filtrations are defined on the generators:

$$
\begin{gathered}
\operatorname{deg}(y)=1, \quad \operatorname{deg}(x)=\operatorname{deg}(\alpha)=0 \\
\operatorname{deg}\left(\frac{\partial}{\partial x}\right)=1, \quad \operatorname{deg}(x)=0 \\
\operatorname{deg}\left(\frac{\partial}{\partial A_{i, i+1}}\right)=1, \quad \operatorname{deg}\left(A_{i, i+1}\right)=0, \quad i=0, \ldots, \ell-1 .
\end{gathered}
$$

It is easy to see that we have

$$
\begin{gathered}
g r H_{1}(c)=\mathbb{Z}_{\ell} \ltimes \mathbb{C}[x, y], \quad \operatorname{gr} \mathrm{D}\left(\mathfrak{t}^{r e g}\right)=\mathbb{C}\left[x^{ \pm 1}, y\right], \\
\operatorname{gr} \mathrm{D}\left(R Q_{n}\right)=\mathbb{C}\left[A_{0,1}, \ldots, A_{\ell-1,0}, B_{1,0}, \ldots, B_{0, \ell-1}\right],
\end{gathered}
$$

where $B_{i+1, i}$ is the image of $\frac{\partial}{\partial A_{i, i+1}}, i=0, \ldots, \ell-1$.

The maps $\mathfrak{R}_{c}^{t w}$ and $\Theta_{c}$ respect the filtrations. Obviously, the associated graded map $g r \Theta_{c}^{s p h}$ is just the inclusion $\mathbb{C}[x, y]^{\mathbb{Z}_{\ell}} \hookrightarrow \mathbb{C}\left[x^{ \pm 1}, y\right]$. The map $g r \Re_{c}^{t w}$ is the restriction map: $\left.f \mapsto f\right|_{\mathcal{S}}$, where $\mathcal{S}=\left\{A_{0,1}=\cdots=A_{\ell-1,0}, B_{1,0}=\cdots=B_{0, \ell-1}\right\}$. We have $\operatorname{Im} g r \Re_{c}^{t w}=\operatorname{Im} g r \Theta_{c}^{s p h}$ by the easiest case of the main theorem from [Ga].

Let us remark that we have $I_{c} \subset \operatorname{ker} \Re_{c}^{t w}$ where $I_{c}$ is the ideal generated by the elements $A_{i, i+1} \frac{\partial}{\partial A_{i, i+1}}-A_{i-1, i} \frac{\partial}{\partial A_{i-1, i}}-C_{i} i=0, \ldots, \ell-1$. Moreover it is easy see that $g r I_{c}=\operatorname{ker} g r \mathfrak{R}_{c}^{t w}$. Hence we have $g r \operatorname{Im} \mathfrak{R}_{c}^{t w}=\operatorname{Im} g r \mathfrak{R}_{c}^{t w}$ because we have $g r$ ker $\mathfrak{R}_{c}^{t w} \supset g r I_{c}=\operatorname{ker} g r \mathfrak{R}_{c}^{t w}$ and $g r$ ker $\mathfrak{R}_{c}^{t w} \subset \operatorname{ker} g r \mathfrak{R}_{c}^{t w}$ for the obvious reasons. Obviously, $g r \operatorname{Im} \Theta_{c}=\operatorname{Im} g r \Theta_{c}$ (because both maps are injective) hence we get $g r \operatorname{Im} \Theta_{c}=g r \operatorname{Im} \Re_{c}^{t w}$. Together with the inclusion $\operatorname{Im} \Theta_{c}^{s p h} \subset \operatorname{Im} \mathfrak{R}_{c}^{t w}$ this completes the proof.

Proof of the theorem. From the paper [EG] we know that $\mathfrak{R}_{k}^{t w}\left(\mathrm{D}\left(\mathfrak{g l}_{n}\right)^{\mathfrak{p g}_{n}}\right)=$ $\Theta_{k}\left(\mathrm{e} H_{n}(k) \mathrm{e}\right)$, hence Lemma 3.1 implies that $\mathfrak{R}_{k, c}^{t w}\left(\mathrm{D}\left(\widetilde{R Q_{n}}\right)^{\mathfrak{p} \mathfrak{g}_{n}}\right)=$ $\Theta_{k, c}\left(\left(\mathrm{e} H_{n}(k, c) \mathrm{e}\right)_{\delta_{\Gamma}}\right)$.

On the other hand the map $i^{*}$ from Lemma 3.2 can be shown to be surjective. The proof is the induction by the order of the differential operators from $\mathrm{D}^{0}$. In particular, the image of $i^{*}$ contains the operators $d_{m}:=\sum_{i=1}^{n} \frac{\partial^{m}}{\partial\left(A_{0,1}\right)_{i i}^{m}} \cdots \frac{\partial^{m}}{\partial\left(A_{\ell-1,0}\right)_{i i}^{m}}$. The same computation as in the proposition 3.3 implies that $\mathfrak{R}_{c}^{t w}\left(d_{m}\right)=\Theta_{0, c}^{s p h}\left(\sum_{i=1}^{n} y_{i}^{\ell m}\right)$. That imply $\mathfrak{R}_{k, c}\left(\mathrm{D}\left(R Q_{n}^{0}\right)\right) \supset \Theta_{k, c}\left(\left(\mathrm{e} H_{n}(k, c) \mathrm{e}\right)_{\delta}\right)$ because the algebra $\mathrm{e} H_{n}(k, c) \mathrm{e}$ is generated by $\sum_{i=1}^{n} x_{i}^{p \ell}$ and $\sum_{i=1}^{n} y_{i}^{\ell q}, p, q \geq 0$.

The slight modification of the argument from the proposition 3.3 proves that we actually have equality $\mathfrak{R}_{k, c}\left(\mathrm{D}\left(R Q_{n}^{0}\right)\right)=\Theta_{k, c}\left(\left(\mathrm{e} H_{n}(k, c) \mathrm{e}\right)_{\delta}\right)$. But $\mathrm{e} H_{n}(k, c)$ e is free as $\mathbb{C}\left[x_{1}, \ldots, x_{n}\right]$-module (under the left multiplication), hence we have

$$
\left(\mathrm{e} H_{n}(k, c) \mathrm{e}\right)_{\delta_{\Gamma}} \cap\left(\mathrm{e} H_{n}(k, c) \mathrm{e}\right)_{\delta}=\mathrm{e} H_{n}(k, c) \mathrm{e} .
$$

Thus we proved the theorem. 


\section{Cyclotomic Bessel function}

4.1. Definitions. For $P \in \mathbb{C}\left[y_{1}, \ldots, y_{n}\right]^{\Gamma_{n}}$ let us define a $\Gamma_{n}$-invariant differential operator $\mathcal{D}_{P}:=\Theta_{k, c}^{s p h}(P)=P\left(\mathcal{D}_{1}, \ldots, \mathcal{D}_{n}\right)$. The differential operators $\mathcal{D}_{P}, P \in$ $\mathbb{C}\left[y_{1}, \ldots, y_{n}\right]^{\Gamma_{n}}$ mutually commute and we can study their common eigenfunctions. The problem makes sense if we replace $\Gamma_{n}$ by any complex reflection group $W$ and $D_{i}$ by the corresponding Dunkl operators [DO]. In the case when $W$ is a Coxeter group the problem was studied by $[\mathrm{Op}]$. Most of the proofs from $[\mathrm{Op}]$ are valid in the case when $W$ is a complex reflexion group. Below we refer to these proofs.

Let us define some simply connected domain $\mathcal{C}$ inside $\mathfrak{t}^{\text {reg }}$. For that we define the set of cuts of $\mathfrak{t}^{\text {reg }}$ :

$$
\begin{aligned}
\operatorname{cut}_{i} & =\left\{x \in \mathfrak{t}^{r e g} \mid \Re x_{i}=0, \Im x_{i}>0\right\}, \\
\operatorname{cut}_{i, j ; m} & =\left\{x \in \mathfrak{t}^{r e g} \mid \Re\left(x_{i} / x_{j}-e^{2 \pi \sqrt{-1} m / \ell}\right)\right. \\
& \left.=0, \Im\left(x_{i} / x_{j}-e^{2 \pi \sqrt{-1} m / \ell}\right)>0\right\}, \quad 0 \leq m<\ell^{\prime}, \\
\operatorname{cut}_{i, j ; m} & =\left\{x \in \mathfrak{t}^{r e g} \mid \Re\left(x_{i} / x_{j}-e^{2 \pi \sqrt{-1} m / \ell}\right)\right. \\
& \left.=0, \Im\left(x_{i} / x_{j}-e^{2 \pi \sqrt{-1} m / \ell}\right)<0\right\}, \quad \ell^{\prime} \leq m<\ell,
\end{aligned}
$$

where $1 \leq i<j \leq n$ and $\ell^{\prime}=\ell / 2$ if $\ell$ is even, $\ell^{\prime}=(\ell-1) / 2$ if $\ell$ is odd. Let cuts $:=\cup_{1 \leq i<j \leq n} \cup_{0 \leq m<\ell}$ cut $_{i j ; m} \cup \cup_{1 \leq i \leq n}$ cut $_{i}$ and $\mathcal{C}=\mathfrak{t}^{\text {reg }} \backslash$ cuts.

Let us fix $\lambda \in \mathbb{C}^{n}$. It is easy to see that the space $V_{\lambda}^{k, c}$ of solutions of the system of equations in the domain $\mathcal{C}$ :

$$
\mathcal{D}_{P} f=P(\lambda) f, \quad \forall P \in \mathbb{C}\left[y_{1}, \ldots, y_{n}\right]^{\Gamma_{n}},
$$

has finite dimension (it is actually equal to $\left|\Gamma_{n}\right|$ for generic $\lambda$ (see [Op], Corollary 3.7)).

A function $f \in V_{\lambda}^{k, c}$ is analytic in $\mathcal{C}$ and can be analytically continued to $\mathfrak{t}^{r e g}$ but the continued function is multivalued because $\mathfrak{t}^{\text {reg }}$ is not simply connected. Moreover, we can continue $f \in V_{\lambda}^{k, c}$ to $\mathfrak{t}$ if we allow the singularities. Let us denote this continuation by $\tilde{f}$.

Let us assume that $k, C_{i} \in \mathbb{R}, 0 \leq i \leq \ell-1$ (the relation between $C$ and $c$ was explained at subsection 2.4). To simplify the exposition we also assume that $k>0$, $\sum_{i=0}^{s} C_{i} \geq 0,0 \leq s<\ell$. The general case can be treated similarly.

The functions $\tilde{f}, f \in V_{\lambda}^{k, c}$ could have the singularities. Let us assume that $C_{i} \notin \mathbb{Z}$, $i=1, \ldots, \ell-1$. Then the local analysis shows (see section 7 of $[\mathrm{Op}]$ ) that for any $j$, $1 \leq j \leq n$ and $f \in V_{\lambda}^{k, c}$ we can present $\tilde{f}$ in the form

$$
\tilde{f}(x)=\sum_{s \in S} x_{j}^{a_{s}} F_{s}(x),
$$

where $S$ is a subset of $\{0, \ldots, \ell-1\}, a_{s}$ are defined at the proof of the proposition 3.3 and $F_{s}, s \in S$ is a nonzero function analytic at the generic point of the divisor $\left\{x_{j}=0\right\}$. In the case when the assumption on $C$ does not hold there is a similar presentation for $\tilde{f}$ (see section 7 of $[\mathrm{Op}]$ ) which involves the logothimic functions.

Let us assume that $k \notin \mathbb{Z}+\frac{1}{2}$. Then it is also possible to show (see section 7 of [Op]) that for any $i, j, m, 1 \leq i \neq j \leq n, 0 \leq m<\ell$ and $f \in V_{\lambda}$ we can present $\tilde{f}$ in 
the form

$$
\tilde{f}(x)=\sum_{s \in S}\left(x_{i}-\epsilon^{m} x_{j}\right)^{2 s} F_{s}(x)+\sum_{t \in T}\left(x_{i}-\epsilon^{m} x_{j}\right)^{2 k+1+2 t} G_{t}(x),
$$

where $S, T$ are subsets of $\left\{0,1, \ldots, \ell^{\prime}\right\}$ and $F_{s}, G_{t}, s \in S, t \in T$ are non zero functions analytic at the generic point of the divisor $\left\{x_{i}-\epsilon^{m} x_{j}=0\right\}$. Again if $k \in \mathbb{Z}+\frac{1}{2}$ then there is a similar expression for $\tilde{f}$ which involves the logorithmic functions.

Let us also remark that the real part $\mathfrak{t}_{\mathbb{R}}^{r e g}$ of $\mathfrak{t}^{\text {reg }}$ is a subset of $\mathcal{C}$. Hence the restriction $\left.f\right|_{\mathfrak{t}_{\mathbb{R}}^{\text {reg }}}$ is a well defined single valued function. Let us also denote by $\Lambda^{\text {reg }}$ the subset of $\mathbb{C}^{n}$ such that $\lambda \in \Lambda^{r e g}$ if and only if $\lambda_{i} \neq 0, \lambda_{i}^{\ell} \neq \lambda_{j}^{\ell}, 1 \leq i \neq j \leq n$.

Definition. The cyclotomic Bessel function $B_{\lambda}^{k, c}, \lambda \in \Lambda^{r e g}$ is a function from $V_{\lambda}^{k, c}$ such that

(1) $\tilde{B}_{\lambda}^{k, c}$ has no singularities (i.e. takes only finite values) on $\mathfrak{t} \simeq \mathbb{C}^{n}$,

(2) $\left.B_{\lambda}^{k, c}\right|_{\mathfrak{t}_{\mathbb{R}}^{r e g}}$ is $S_{n}$-invariant,

(3) $\tilde{B}_{\lambda}^{k, c}(0)=1$.

According to propositions 5.6, 6.8 and corollary 7.16 of [Op] the conditions (1), (2), (3) define the function $B_{\lambda}^{k, c}$ uniquelly. Moreover, from the results of [Op] it follows that the function $\tilde{B}_{\lambda}^{k, c}$ is a singlevalued function.

Remark 4.1. From the assumptions on the parameters $C_{i}, 0 \leq i<\ell$ we see that $a_{i}<a_{\ell-1}=0,0 \leq i<\ell-1$. Hence the generic function from $V_{\lambda}^{k, c}$ has singularities.

Remark 4.2. When $n=1$ and $\ell=2$ the function $B_{\lambda}^{k, c}$ is related to the classical Bessel function $J_{C_{1}}$ by the formula $(\lambda x)^{C_{1}} B_{\lambda}^{k, c}(x) / \Gamma\left(C_{1}\right)=J_{C_{1}}(2 x \lambda)$.

Using the theory of the deformed Harish-Chandra homomorphism we find a Kirillov-type $[\mathrm{K}]$ integral formula for the Bessel function. We use the ideology of the paper $[\mathrm{EFK}]$ to do that.

Let $\mathbf{K}_{n}=U(n)^{\times \ell} \subset G_{n}$ be the maximal compact subgroup of $G_{n}$. Let $Q^{o p}$ be a cyclic quiver with $\ell$ vertices and the edges oriented counterclockwise. Let $R Q_{n}^{o p}$ be the space of representations of $\mathbb{C} Q^{o p}$ of dimension $n \delta$. Let us denote by $\Delta^{o p}$ the map which sends the element $y \in \mathbb{C}^{n}$ to the element $(\operatorname{diag}(y), \ldots, \operatorname{diag}(y)) \in R Q_{n}^{o p}$. Let us denote by $\mathcal{O}_{\lambda} \subset R Q_{n}^{o p}$ the $\mathbf{K}_{n}$-orbit of the element $\Delta^{o p}(\lambda)$. The invariant measure on $\mathbf{K}_{n}$ induces a $\mathbf{K}_{n}$-invariant measure $d \mu_{\lambda}$ on $\mathcal{O}_{\lambda}$. Then the space $V_{\lambda}:=L^{2}\left(\mathcal{O}_{\lambda}\right)$ has a natural structure of $\mathbf{K}_{n}$-module:

$$
(g \cdot f)(B)=f\left(g^{-1} \cdot B\right) .
$$

The action of $\mathbf{K}_{n}$ respects the Hermitian product $\langle f, g\rangle_{\lambda}:=\int_{\mathcal{O}_{\lambda}} f(A) \bar{g}(A) d \mu_{\lambda}(A)$ on $V_{\lambda}$.

4.2. Now let us assume that $C \in \mathbb{Z}^{\ell}$ and $k \in \mathbb{Z}_{+}$. Then the map $\rho_{k, c}$ gives us the representation of $\mathbf{K}_{n}$ which we denote by $W_{k, c}$ (it is isomorphic to $W_{k}$ as a vector space). Let $W_{k, c}^{\vee}$ be its dual. The vector space $V_{\lambda}$ is a unitary representation of $\mathbf{K}_{n}$ and we have:

Proposition 4.1. If $\lambda \in \Lambda^{\text {reg }}$ then there exists a unique up to scaling injective map of $\mathbf{K}_{n}$-representations $\eta_{k, c}^{\lambda}: W_{k, c}^{\vee} \rightarrow V_{\lambda}$. 
Proof. Because of the conditions on $\lambda$ we have $\mathcal{O}_{\lambda} \simeq \mathbf{K}_{n} / \mathbf{T}_{n}$ where $\mathbf{T}_{n}$ is a torus which is equal to the stabilizer of $\Delta^{o p}(\lambda)$. On the other hand the space $L^{2}\left(\mathbf{K}_{n}\right)$ has the left and right actions of $\mathbf{K}_{n}$ and by the Peter-Weyl theorem it decomposes into the direct sum of subrepresentations

$$
L^{2}\left(\mathbf{K}_{n}\right)=\hat{\oplus}_{V \in \hat{\mathbf{K}}_{n}} V^{\vee} \otimes V,
$$

where $\hat{\mathbf{K}}_{n}$ is a notation for the space of all irreducible representations of $\mathbf{K}_{n}$ and $\hat{\oplus}$ is a notation for the completed direct sum. Hence we have

$$
L^{2}\left(\mathcal{O}_{\lambda}\right)=\hat{\oplus}_{V \in \widehat{\mathbf{K}}_{n}} V^{\vee} \otimes(V)^{\mathbf{T}_{n}} .
$$

To finish the proof let us notice that $W_{k, c}^{\mathbf{T}_{n}} \simeq \mathbb{C}$.

4.3. Integral formula. From the explicit construction of $W_{k, c} \subset \mathbb{C}\left[y_{1}, \ldots, y_{n}\right]$ we know that the monomials $y_{1}^{i_{1}} \ldots y_{n}^{i_{n}}, \sum_{j=1}^{n} i_{j}=n k$ span $W_{k, c}$ and $W_{k, c}[0]=$ $\left\langle\left(y_{1} \ldots y_{n}\right)^{k}\right\rangle$. Let us define the function $m_{k}$ on $U(n)$ by the formula:

$g\left(\left(y_{1} \ldots y_{n}\right)^{k}\right)=m_{k}(g)\left(y_{1} \ldots y_{n}\right)^{k}+$ the linear combination of the other monomials.

Then we have

Corollary 1. For $\lambda \in \Lambda^{\text {reg }}$ we have

$$
B_{\lambda}^{k, c}(x) \sim \frac{\int_{\mathbf{K}_{n}} e^{\operatorname{tr}\left(g \Delta^{o p}(\lambda) g^{-1} \Delta(x)\right)} m_{k}\left(g_{0}\right) \prod_{i=0}^{\ell-1} \operatorname{det}\left(g_{i}\right)^{C_{i}} d \mu_{l}(g)}{\delta_{k, c}(x)},
$$

where $d \mu_{l}$ is a left invariant measure on $\mathbf{K}_{n}$ and $\sim$ stands for being proportional.

Before giving the prove let us discuss the formula (1) in the case $n=1$.

4.4. Case $\mathbf{n}=1$. In this case we can omit $k$ from the notations. The space $V_{\lambda}^{c}$ is a space of solutions of the ODE:

$$
\left(\frac{\partial}{\partial x}+\frac{\tilde{C}_{0}}{x}\right)\left(\frac{\partial}{\partial x}+\frac{\tilde{C}_{0}+\tilde{C}_{1}}{x}\right) \ldots\left(\frac{\partial}{\partial x}+\frac{\tilde{C}_{0}+\cdots+\tilde{C}_{\ell-1}}{x}\right) f=\lambda^{l} f .
$$

Because of the assumption on the parameters $C_{i}, 0 \leq i<\ell$ we know that $a_{i}<$ $a_{\ell-1}=0,0 \leq i<\ell$. Hence the space of the function from $V_{\lambda}^{c}$ without a pole at $x=0$ is one-dimensional and spanned by the Bessel function $B_{\lambda}^{c}$.

Corollary gives the formula:

$$
B_{\lambda}^{c}(x) \sim x^{-\sum_{s=0}^{\ell-1} s C_{s}} \int_{[0,1)^{\ell}} e^{\sum_{j=0}^{\ell-1} 2 \pi \sqrt{-1} \varphi_{j} C_{j}+e^{2 \pi \sqrt{-1}\left(\varphi_{j}-\varphi_{j+1}\right)} \lambda x} d \varphi_{0} \ldots d \varphi_{\ell-1},
$$

where we assume that $\varphi_{\ell}=\varphi_{0}$.

Proof of Corollary for $n=1$. It is easy to check that RHS of (2) is a function from $V_{\lambda}^{c}$. Let us explain why it has no pole at $x=0$. That is we need to prove that the integral in (2) has the zero of order $\sum_{s=0}^{\ell-1} s C_{s}$ at $x=0$.

Let us denote the integrand in (2) by $F(\varphi, x)$. Let $e_{i}, i=0, \ldots, \ell-1$ be a standard basis in $\mathbb{Z}^{\ell}$ and $\nu_{i}=e_{i}-e_{i+1}, 0 \leq i<\ell-1, \nu_{\ell-1}=e_{\ell-1}-e_{0}$. Then we have:

$$
\frac{\partial^{t}}{\partial x^{t}} F(0, \varphi)=\lambda^{t} \sum_{i_{1}, \ldots, i_{t}=0}^{\ell-1} e^{\left(C-\sum_{s=1}^{t} \nu_{i_{s}}, \varphi\right)}
$$


where $(y, \varphi)=2 \pi \sqrt{-1} \sum_{i=0}^{\ell-1} y_{i} \varphi_{i}$. Only terms such that $C-\sum_{s=1}^{t} \nu_{i_{s}}=0$ give a nonzero input into the integral $\int_{[0,1)^{\ell}} \frac{\partial^{t}}{\partial x^{t}} F(0, \varphi) d \varphi_{0} \ldots d \varphi_{\ell-1}$. There are no such terms if $t<\sum_{s=0}^{\ell-1} s C_{s}$. Moreover, if $t=\sum_{s=0}^{\ell-1} s C_{s}$ then there is only one such term.

Corollary 2. For $\lambda \neq 0$ we have

$$
B_{\lambda}^{c}(x)=\frac{t !}{(\lambda x)^{t}} \int_{[0,1)^{\ell}} e^{\sum_{j=0}^{\ell-1} 2 \pi \sqrt{-1} \varphi_{j} C_{j}+e^{2 \pi \sqrt{-1}\left(\varphi_{j}-\varphi_{j+1}\right)} \lambda x} d \varphi_{0} \ldots d \varphi_{\ell-1},
$$

where $t=\sum_{s=0}^{\ell-1} s C_{s}$.

\section{5.}

Proof of the Corollary 1. One can show that we actually have $\operatorname{Im} \eta_{k, c}^{\lambda} \subset C^{\infty}\left(\mathcal{O}_{\lambda}\right)$. Let us choose a nonzero function $\varphi_{\lambda}^{k, c} \in \eta_{k, c}^{\lambda}\left(W_{k}^{\vee}[0]\right) \in C^{\infty}\left(\mathcal{O}_{\lambda}\right)$ where the space $W_{k}[0]^{\vee} \subset W_{k, c}$ is the one defined in subsection 4.3. Then it is easy to see that RHS of (1) is proportional to the function:

$$
h_{\lambda}(x)=\frac{\int_{\mathcal{O}_{\lambda}} \varphi_{\lambda}^{k, c}(A) e^{t r(A \Delta(x))} d \mu_{\lambda}(A)}{\delta_{k, c}(x)} .
$$

The space $W_{k, c}^{\vee}$ is a subspace of $V_{\lambda}$ and we can choose a basis $v_{1}^{\vee}, \ldots, v_{N}^{\vee}$ in $W_{k, c}^{\vee}$ and $v_{1}, \ldots, v_{N}$ be its dual basis. Let us define the vector valued function

$$
f_{\lambda}(B)=\sum_{i=1}^{N} v_{i} \int_{\mathcal{O}_{\lambda}} v_{i}^{\vee}(A) \exp (\operatorname{tr}(A B)) d \mu_{\lambda}(A) .
$$

It is easy to see that $f_{\lambda} \in F u n_{k, c}$ and $\left.f_{\lambda}\right|_{\mathfrak{t}}$ is proportional to $h_{\lambda}$. For every $P \in$ $\mathbb{C}\left[y_{1}, \ldots, y_{n}\right]^{\Gamma_{n}}$ there exists $G_{n}$-invariant function $S_{P} \in \mathbb{C}\left[R Q_{n}^{o p}\right]^{P G_{n}}$ such that

$S_{P}\left(\Delta^{o p}(y)\right)=P(y)$. One should think of $S_{P}$ as a differential operator on $R Q_{n}$ with constant coefficients. From the proof of the main theorem one can see that $\mathfrak{R}_{k, c}^{t w}\left(S_{P}\right)=$ $\mathcal{D}_{P}$. Also from the formula for $f_{\lambda}$ one can see that $S_{P} f_{\lambda}=S_{P}\left(\Delta^{o p}(\lambda)\right) f_{\lambda}=P(\lambda) f_{\lambda}$. Finally we use that $f_{\lambda}(\Delta(x)) \in W_{k}[0]$ for all $x \in \mathbb{C}^{n}$.

Let us prove that RHS of (1) is analytic. From the discussion before the definition of the Bessel function we see that the functions from $V_{\lambda}^{k, c}$ can have the poles only along the divisors $x_{j}=0, j=1, \ldots, n$. Hence we only need to show that the order of vanishing of the integral in RHS of (1) is at least $\sum_{s=0}^{\ell-1} s C_{s}$. Let us show it for $j=1$.

Indeed, every element $g \in \mathbf{K}_{n}$ can be uniquely presented in the form $g=\tilde{g} A(\varphi)$, where $\tilde{g} \in S U(n)^{\times \ell}$ and $\varphi \in[0,1)^{\ell}, A_{i}=\operatorname{diag}\left(e^{2 \pi \sqrt{-1} \varphi_{i}}, 1, \ldots, 1\right), 0 \leq i<\ell$. For $\varphi \in[0,1)^{\ell}, x \in \mathbb{C}^{n}$ let $\Delta(x, \varphi)$ be the element of $R Q_{n}$ such that $\Delta(x, \varphi)_{i, i+1}=$ $\operatorname{diag}\left(e^{2 \pi \sqrt{-1}\left(\varphi_{i}-\varphi_{i+1}\right)} x_{1}, x_{2}, \ldots, x_{n}\right), 0 \leq i<\ell$. We can rewrite the integral from (1) in the form:

$$
\text { Int }=\int_{\varphi \in[0,1)^{\ell}} e^{(\varphi, C)}\left(\int_{\tilde{G}_{n}} e^{\operatorname{tr}\left(\tilde{g} \Delta^{o p}(\lambda) \tilde{g}^{-1} \Delta(x, \varphi)\right)} m_{k}\left(\tilde{g}_{0}\right) d \tilde{\mu}_{l}(\tilde{g})\right) d \varphi_{0} \ldots d \varphi_{\ell-1},
$$

where $\tilde{G}_{n}=S U(n)^{\times \ell}$ and $d \tilde{\mu}_{l}$ is the corresponding left $\tilde{G}_{n}$-invariant measure. Now let us notice that when $x_{1}=0$ the integral:

$$
\int_{\tilde{G}_{n}} e^{\operatorname{tr}\left(\tilde{g} \Delta^{o p}(\lambda) \tilde{g}^{-1} \Delta(x, \varphi)\right)} m_{k}(\tilde{g}) d \tilde{\mu}_{l}(\tilde{g})
$$


does not depend of $\varphi$. Hence the computation of the order of vanishing of Int at $x_{1}=0$ is essentially the same as in the case $n=1$.

Remark 4.3. It is easy to see that the integral in the formula (1) can be reduced to the integral over $\mathbf{K}_{n} / \mathbf{T}_{n}$ because the integrand is $\mathbf{T}_{n}$-invariant.

4.6. An interpretation in term of spherical functions. Let us illuminate the connection with the theory of spherical functions and Kirillov's orbit method. Let $R Q_{n}(\mathbb{R}) \subset R Q_{n}\left(R Q_{n}^{o p p}(\mathbb{R}) \subset R Q_{n}^{o p p}\right)$ be the subspace of the real points of $R Q_{n}$ $\left(R Q_{n}^{\text {opp }}\right)$. Let $G_{n}(\mathbb{R})$ be the real part of $G_{n}$. As $G_{n}(\mathbb{R})$ acts on the space $R Q_{n}(\mathbb{R})$, we have the group $\mathbf{H}_{n}(\mathbb{R})=R Q_{n}(\mathbb{R}) \rtimes G_{n}(\mathbb{R})$ with the relations:

$$
(A, g) \cdot(B, h)=(A+g \cdot B, g h),
$$

where $A, B \in R Q_{n}(\mathbb{R})$ and $g, h \in G_{n}(\mathbb{R})$.

The group $\mathbf{H}_{n}(\mathbb{R})$ has a left and right action of the subgroup $G_{n}(\mathbb{R})$. In particular, the space $F u n_{k, c}$ is the space of $W_{k, c}$-valued functions on $\mathbf{H}_{n}(\mathbb{R})$ which are invariant with respect to the left action of $G_{n}(\mathbb{R})$ and $G_{n}(\mathbb{R})$-equivariant with respect to the right action. Hence from the ideology of the paper [EFK] we know that we can construct the functions from Fun $_{k, c}$ starting from the representations of $\mathbf{H}_{n}(\mathbb{R})$.

Now we construct unitary representations of $\mathbf{H}_{n}(\mathbb{R})$. Let $\mathcal{O}_{\lambda}^{\mathbb{R}}, \lambda \in \mathbb{R}^{n}, \lambda_{i} \neq \lambda_{j}$, $1 \leq i<j \leq n$ be the $G_{n}(\mathbb{R})$-orbit of $\Delta^{o p}(\lambda)$ inside $R Q_{n}(\mathbb{R})$. Let $d \mu_{\lambda}$ be a left $G_{n}(\mathbb{R})$-invariant measure on $\mathcal{O}_{\lambda}^{\mathbb{R}}$. Then the space $V_{\lambda}^{\mathbb{R}}:=L^{2}\left(\mathcal{O}_{\lambda}^{\mathbb{R}}\right)$ has natural Hermitian product: $\langle f, g\rangle_{\lambda}=\int_{\mathcal{O}_{\lambda}} f(A) \bar{g}(A) d \mu_{\lambda}(A)$, and the group $\mathbf{H}_{n}(\mathbb{R})$ acts by unitary operators on $V_{\lambda}^{\mathbb{R}}$ :

$$
((0, g) \cdot f)(A)=f\left(g^{-1} \cdot A\right), \quad((B, 1) \cdot f)(A)=\exp (2 \pi \sqrt{-1} \operatorname{tr}(A B)) f(A),
$$

where $g \in G_{n}(\mathbb{R}), A \in R Q_{n}(\mathbb{R})$.

Let $\tilde{V}_{\lambda}^{\mathbb{R}}$ be the space of smooth functions on $\mathcal{O}_{\lambda}^{\mathbb{R}}$. The complexifications of the groups $G_{n}(\mathbb{R})$ and $\mathbf{K}_{n}$ coincide. Hence Proposition 4.1 implies that there exists a unique up to scaling map of $G_{n}(\mathbb{R})$-representations $\eta_{k, c}^{\lambda}: W_{k, c}^{\vee} \rightarrow \tilde{V}_{\lambda}^{\mathbb{R}}$, where $W_{k, c}^{\vee}$ is a representation of $G_{n}(\mathbb{R})$ dual to $W_{k, c}$.

Clearly the image of $\eta_{k, c}^{\lambda} \subset \tilde{V}_{\lambda}^{\mathbb{R}}$ does not lie inside $V_{\lambda}^{\mathbb{R}}$ but we can use pairing $\langle\cdot, \cdot\rangle_{\lambda}$ for the elements of $\tilde{V}_{\lambda}^{\mathbb{R}}$ if we think of the integration over $\mathcal{O}_{\lambda}$ in the sense of generalized functions. Keeping it in mind we can write the formula for the spherical function:

$$
f_{\lambda}^{\mathbb{R}}(X)=\sum_{i=1} v_{i}\left\langle\eta_{k, c}^{\lambda}\left(v_{i}^{\vee}\right), X \cdot 1\right\rangle_{\lambda}
$$

where $X \in \mathbf{H}_{n}(\mathbb{R}), v_{1}, \ldots, v_{N}$ is a basis in $W_{k, c}$ and $v_{1}^{\vee}, \ldots, v_{n}^{\vee}$ is a dual basis. It is clear that we have

Corollary 3. For $\lambda \in \mathbb{R}^{n}$ such that $\lambda_{i} \neq \lambda_{j}, 1 \leq i \neq j \leq n$ we have

$$
f_{\lambda}^{\mathbb{R}}\left(\left(\Delta^{o p p}(x), 1\right)\right) \sim B_{\lambda}(2 \pi \sqrt{-1} x),
$$

where $\sim$ stands for being proportional.

When $\ell=1$ then the coadjoint orbit $\mathcal{O}_{\lambda}^{\mathbb{R}}$ corresponds to the principal series representation $\mathcal{P}^{\lambda}$ of $G L_{n}(\mathbb{R})$ (see the User's Guide in $\left.[K]\right)$. For $k=0$ the function $f_{\lambda}^{\mathbb{R}}((1, A)), A \in \mathfrak{g l}_{n}(\mathbb{R})$ is the generalized $\mathfrak{g l}_{n}(\mathbb{R})$-character of $\mathcal{P}^{\lambda}$ and the formula (3) is the classical Kirillov's integral formula for the character. For the general $k$, 
the construction is a degeneration of the construction of $[\mathrm{EFK}]$ where the vector valued characters of $G L(n, \mathbb{C})$ were studied. Indeed, the $W_{k}$-valued character of $G=G L(n, \mathbb{C})$ can be interpreted as function on $G \times G$ which is left $G$-invariant and right $G$-equivariant (for more detailed discussion see [Op]). While we degenerate the group $G \times G$ into $\mathfrak{g l}(n, \mathbb{C}) \rtimes G L(n, \mathbb{C})$ we see that the construction for the Jack polynomials from $[\mathrm{EFK}]$ give us the construction for the Bessel function from this note.

In the paper $[\mathrm{Ob}]$ we study the space of the $W_{k}$-valued functions on $G L(2 n, \mathbb{C})$ which are invariant with respect to the left action of $G L(n, \mathbb{C}) \times G L(n, \mathbb{C})$ and $W_{k, c^{-}}$ equivariant with respect to the right action of $G L(n, \mathbb{C}) \times G L(n, \mathbb{C})$. In $[\mathrm{Ob}]$ we use representation theory of $G L(2 n, \mathbb{C})$ to construct the Macdonald-Koornwinder polynomials. If we degenerate $G L(2 n, \mathbb{C})$ into the group $\mathfrak{g l}(n, \mathbb{C})^{\oplus 2} \rtimes G L(n, \mathbb{C})^{\times 2}$, we get Corollary 1 for $\ell=2$. Indeed, the group $\mathfrak{g l}(n, \mathbb{C})^{\oplus 2} \rtimes G L(n, \mathbb{C})^{\times 2}$ is the complexification of the group $\mathbf{H}_{n}(\mathbb{R})$ and the right and left action of $G L(n, \mathbb{C})^{\times 2}$ on $G L(2 n, \mathbb{C})$ degenerates into the right and left action of $G L(n, \mathbb{C})^{\times 2}$ on the complexification of the group $\mathbf{H}_{n}(\mathbb{R})$.

\section{Acknowledgements}

I am very grateful to my advisor Pavel Etingof for the formulation of the problem and many fruitful discussions which led me to the solution. I want to thank Wee Liang Gan for useful discussions and explaining his results with Victor Ginzburg. I also want to thank Iain Gordon for the attention to the work and pointing out the misprints in the preliminary version of the note. I want to thank Richard Vale for finding a problematic place in the first version of the proof. Finally, I am very grateful to Jasper Stokman for reading the note and giving many useful references.

\section{References}

[BEG] Yu. Berest, P. Etingof, V. Ginzburg, Cherednik algebras and differential operators on quasiinvariants, Duke Math. J. 118, no. 2 (2003), 279-337.

[BEG1] Yu. Berest, P. Etingof, V. Ginzburg, Finite dimensional representations of rational Cherednik algebras, IMRN, no. 19 (2003), 1053-1088.

[Ch] I. Cherednik, Double affine Hecke algebras, KZ equations, and Macdonald operators, IMRN, no. 9 (1992), 171-180.

[DO] C. Dunkl, E. Opdam, Dunkl operators for complex reflection groups. Proc. London Math. Soc., 86, no. 3 (2003) 70-108.

[EFK] P.I.Etingof, I.B.Frenkel, A.A.Kirillov, Jr., Spherical functions on affine Lie group, Duke Math. J., 80, no. 1 (1995), 59-90.

[E] P. Etingof, Cherednik and Hecke algebras of varieties with a finite group action, math.QA/0406499.

[EG] P. Etingof, V. Ginzburg, Symplectic reflection algebras, Calogero-Moser space, and deformed Harish-Chandra homomorphism, math.AG/0011114, Invent. Math., 147, no. 2 (2002), 243-348.

[Ga] W. L. Gan, Chavalley restrictriction theorem for the cyclic quiver, math.RT/0405103.

[GG] W. L. Gan, V. Ginzburg, Almost-commuting variety, D-modules, and Cherednik algebras, math.RT/0409262.

[G] I. Gordon, A remark on rational Cherednik algebras and differential operators on the cyclic quiver, to appear Glasgow Math. Journal, 48 , no. 1 (2006), 145-160.

[Ho] M. Holland, Quantization of the Marsden-Weinstein reduction for extended Dynkin quivers, Ann. Sci. Ecole Norm. Sup., 32 (1999), 813-834.

[K] A. A. Kirillov, Lectures on the orbit Method, Graduate Studies in Mathematics, vol. 64, American Mathematical Society, Providence, R. I. 
[LS] T. Levasseur, J. Stafford, Invariant differential operators and an homomorphism of HarishChandra, J. Amer. Math. Soc. 8, no. 2 (1995), 365-372.

[Ob] A. Oblomkov, Heckman-Opdam's Jacobi polynomials for the $B C_{n}$ root system and generalized spherical functions. Adv. Math. 186 no. 1, (2004), 153-180.

[Op] E.M. Opdam, Bessel functions and the discriminant of a finite Coxeter group. Compositio Math. 85, no. 3 (1993), 333-373.

[V] N. Ja. Vilenkin, N. Ja. Special functions and the theory of group representations, Translations of Mathematical Monographs, Vol. 22 American Mathematical Society, Providence, R. I.

Dpt. of Mathematics, Princeton University, Fine Hall, Washington Road, Princeton NJ 08544 , USA.

E-mail address: oblomkov@math.princeton.edu 